\title{
Evidence of $B^{+} \rightarrow \tau^{+} \nu$ decays with hadronic $B$ tags
}

J.P. Lees, ${ }^{1}$ V. Poireau, ${ }^{1}$ V. Tisserand, ${ }^{1}$ J. Garra Tico, ${ }^{2}$ E. Grauges,${ }^{2}$ A. Palano, ${ }^{3 a, 3 b}$ G. Eigen, ${ }^{4}$ B. Stugu ${ }^{4}$ D. N. Brown, ${ }^{5}$ L. T. Kerth, ${ }^{5}$ Yu. G. Kolomensky, ${ }^{5}$ G. Lynch, ${ }^{5}$ H. Koch, ${ }^{6}$ T. Schroeder, ${ }^{6}$ D. J. Asgeirsson, ${ }^{7}$ C. Hearty, ${ }^{7}$ T. S. Mattison, ${ }^{7}$ J. A. McKenna, ${ }^{7}$ R. Y. So, ${ }^{7}$ A. Khan, ${ }^{8}$ V. E. Blinov, ${ }^{9}$ A. R. Buzykaev, ${ }^{9}$ V.P. Druzhinin, ${ }^{9}$ V. B. Golubev, ${ }^{9}$

E. A. Kravchenko, ${ }^{9}$ A. P. Onuchin, ${ }^{9}$ S. I. Serednyakov, ${ }^{9}$ Yu. I. Skovpen, ${ }^{9}$ E. P. Solodov, ${ }^{9}$ K. Yu. Todyshev, ${ }^{9}$ A. N. Yushkov, ${ }^{9}$ M. Bondioli, ${ }^{10}$ D. Kirkby, ${ }^{10}$ A. J. Lankford ${ }^{10}$ M. Mandelkern,${ }^{10}$ H. Atmacan, ${ }^{11}$ J. W. Gary, ${ }^{11}$ F. Liu, ${ }^{11}$ O. Long, ${ }^{11}$ G. M. Vitug, ${ }^{11}$ C. Campagnari, ${ }^{12}$ T. M. Hong, ${ }^{12}$ D. Kovalskyi, ${ }^{12}$ J. D. Richman, ${ }^{12}$ C. A. West, ${ }^{12}$ A. M. Eisner, ${ }^{13}$ J. Kroseberg, ${ }^{13}$ W. S. Lockman, ${ }^{13}$ A. J. Martinez, ${ }^{13}$ B. A. Schumm, ${ }^{13}$ A. Seiden, ${ }^{13}$ D. S. Chao, ${ }^{14}$ C. H. Cheng, ${ }^{14}$ B. Echenard, ${ }^{14}$ K. T. Flood,${ }^{14}$ D. G. Hitlin, ${ }^{14}$ P. Ongmongkolkul, ${ }^{14}$ F. C. Porter, ${ }^{14}$ A. Y. Rakitin, ${ }^{14}$ R. Andreassen, ${ }^{15}$ Z. Huard, ${ }^{15}$ B. T. Meadows, ${ }^{15}$ M. D. Sokoloff, ${ }^{15}$ L. Sun, ${ }^{15}$ P. C. Bloom, ${ }^{16}$ W. T. Ford, ${ }^{16}$ A. Gaz, ${ }^{16}$ U. Nauenberg, ${ }^{16}$ J. G. Smith, ${ }^{16}$ S. R. Wagner, ${ }^{16}$ R. Ayad, ${ }^{17}{ }^{\dagger}$ W. H. Toki,${ }^{17}$ B. Spaan, ${ }^{18}$ K. R. Schubert, ${ }^{19}$ R. Schwierz, ${ }^{19}$ D. Bernard ${ }^{20}$ M. Verderi, ${ }^{20}$ P. J. Clark ${ }^{21}$ S. Playfer,${ }^{21}$ D. Bettoni, ${ }^{22 a}$ C. Bozzi, ${ }^{22 a}$ R. Calabrese, ${ }^{22 a, 22 b}$ G. Cibinetto, ${ }^{22 a, 22 b}$ E. Fioravanti, ${ }^{22 a, 22 b}$ I. Garzia, ${ }^{22 a, 22 b}$ E. Luppi, ${ }^{22 a, 22 b}$ M. Munerato, ${ }^{22 a, 22 b}$ L. Piemontese, ${ }^{22 a}$ V. Santoro, ${ }^{22 a}$ R. Baldini-Ferroli, ${ }^{23}$ A. Calcaterra, ${ }^{23}$ R. de Sangro, ${ }^{23}$ G. Finocchiaro, ${ }^{23}$ P. Patteri, ${ }^{23}$ I. M. Peruzzi, ${ }^{23, \$}$ M. Piccolo, ${ }^{23}$ M. Rama, ${ }^{23}$ A. Zallo, ${ }^{23}$ R. Contri, ${ }^{24 a, 24 b}$ E. Guido, ${ }^{24 a, 24 b}$ M. Lo Vetere, ${ }^{24 a, 24 b}$ M. R. Monge, ${ }^{24 a, 24 b}$ S. Passaggio, ${ }^{24 a}$ C. Patrignani, ${ }^{24 a, 24 b}$ E. Robutti, ${ }^{24 a}$ B. Bhuyan, ${ }^{25}$ V. Prasad ${ }^{25}$ C. L. Lee, ${ }^{26}$ M. Morii, ${ }^{26}$ A. J. Edwards, ${ }^{27}$ A. Adametz, ${ }^{28}$ U. Uwer, ${ }^{28}$ H. M. Lacker, ${ }^{29}$ T. Lueck, ${ }^{29}$ P. D. Dauncey, ${ }^{30}$ U. Mallik, ${ }^{31}$ C. Chen, ${ }^{32}$ J. Cochran, ${ }^{32}$ W. T. Meyer, ${ }^{32}$ S. Prell, ${ }^{32}$ A. E. Rubin, ${ }^{32}$ A. V. Gritsan, ${ }^{32}$ Z. J. Guo, ${ }^{33}$ N. Arnaud,${ }^{34}$ M. Davier, ${ }^{34}$ D. Derkach,${ }^{34}$ G. Grosdidier, ${ }^{34}$ F. Le Diberder, ${ }^{34}$ A. M. Lutz, ${ }^{34}$ B. Malaescu, ${ }^{34}$ P. Roudeau, ${ }^{34}$ M. H. Schune, ${ }^{34}$ A. Stocchi, ${ }^{34}$ G. Wormser, ${ }^{34}$ D. J. Lange, ${ }^{35}$ D. M. Wright, ${ }^{35}$ C. A. Chavez, ${ }^{36}$ J.P. Coleman, ${ }^{36}$ J. R. Fry, ${ }^{36}$ E. Gabathuler, ${ }^{36}$ D. E. Hutchcroft, ${ }^{36}$ D. J. Payne, ${ }^{36}$ C. Touramanis, ${ }^{36}$ A. J. Bevan, ${ }^{37}$ F. Di Lodovico,${ }^{37}$ R. Sacco, ${ }^{37}$ M. Sigamani, ${ }^{37}$ G. Cowan, ${ }^{38}$ D. N. Brown, ${ }^{39}$ C.L. Davis,${ }^{40}$ A. G. Denig, ${ }^{40}$ M. Fritsch ${ }^{40}$ W. Gradl, ${ }^{40}$ K. Griessinger, ${ }^{40}$ A. Hafner ${ }^{40}$ E. Prencipe, ${ }^{40}$ R. J. Barlow, ${ }^{41,}{ }^{8}$ G. Jackson, ${ }^{41}$ G. D. Lafferty, ${ }^{41}$ E. Behn, ${ }^{42}$ R. Cenci, ${ }^{42}$ B. Hamilton, ${ }^{42}$ A. Jawahery, ${ }^{42}$ D. A. Roberts, ${ }^{42}$ C. Dallapiccola,${ }^{43}$ R. Cowan, ${ }^{44}$ D. Dujmic,${ }^{44}$ G. Sciolla, ${ }^{44}$ R. Cheaib, ${ }^{45}$ D. Lindemann, ${ }^{45}$ P. M. Patel, ${ }^{45, *}$ S. H. Robertson, ${ }^{45}$ P. Biassoni,,${ }^{46 a, 46 b}$ N. Neri, ${ }^{46 a}$ F. Palombo, ${ }^{46 a, 46 b}$ S. Stracka, ${ }^{46 a, 46 b}$ L. Cremaldi, ${ }^{47}$ R. Godang, ${ }^{47, \|}$ R. Kroeger, ${ }^{47}$ P. Sonnek, ${ }^{47}$ D. J. Summers,${ }^{47}$ X. Nguyen, ${ }^{48}$ M. Simard, ${ }^{48}$ P. Taras, ${ }^{48}$ G. De Nardo, ${ }^{49 a, 49 b}$ D. Monorchio, ${ }^{49 a}, 49 b$ G. Onorato, ${ }^{49 a, 49 b}$ C. Sciacca, ${ }^{49 a, 49 b}$ M. Martinelli, ${ }^{50}$ G. Raven,${ }^{50}$ C. P. Jessop, ${ }^{51}$ J. M. LoSecco, ${ }^{51}$ W. F. Wang, ${ }^{51}$ K. Honscheid, ${ }^{52}$ R. Kass, ${ }^{52}$ J. Brau, ${ }^{53}$

R. Frey, ${ }^{53}$ N. B. Sinev, ${ }^{53}$ D. Strom, ${ }^{53}$ E. Torrence, ${ }^{53}$ E. Feltresi, ${ }^{54 a, 54 b}$ N. Gagliardi, ${ }^{54 a, 54 b}$ M. Margoni, ${ }^{54 a, 54 b}$

M. Morandin, ${ }^{54 \mathrm{a}}$ M. Posocco, ${ }^{54 \mathrm{a}}$ M. Rotondo, ${ }^{54 \mathrm{a}}$ G. Simi, ${ }^{54,54 \mathrm{~b}}$ F. Simonetto, ${ }^{54 \mathrm{a}, 54 \mathrm{~b}}$ R. Stroili, ${ }^{54 \mathrm{a}, 54 \mathrm{~b}}$ S. Akar, ${ }^{55}$

E. Ben-Haim, ${ }^{55}$ M. Bomben, ${ }^{55}$ G. R. Bonneaud, ${ }^{55}$ H. Briand, ${ }^{55}$ G. Calderini, ${ }^{55}$ J. Chauveau, ${ }^{55}$ O. Hamon, ${ }^{55}$ Ph. Leruste, ${ }^{55}$ G. Marchiori, ${ }^{55}$ J. Ocariz, ${ }^{55}$ S. Sitt, ${ }^{55}$ M. Biasini, ${ }^{56 a, 56 b}$ E. Manoni,${ }^{56 a, 56 b}$ S. Pacetti, ${ }^{56 a, 56 b}$ A. Rossi, ${ }^{56 a, 56 b}$ C. Angelini, ${ }^{57 a, 57 b}$ G. Batignani, ${ }^{57 a, 57 b}$ S. Bettarini ${ }^{57 a, 57 b}$ M. Carpinelli, ${ }^{57 a, 57 b, \pi l}$ G. Casarosa, ${ }^{57 a, 57 b}$ A. Cervelli, ${ }^{57 a, 57 b}$ F. Forti, ${ }^{57 a, 57 b}$ M. A. Giorgi, ${ }^{57 a, 57 b}$ A. Lusiani, ${ }^{57 a, 57 c}$ B. Oberhof,,${ }^{57 a, 57 b}$ E. Paoloni, ${ }^{57 a, 57 b}$ A. Perez, ${ }^{57 a}$ G. Rizzo, ${ }^{57 a, 57 b}$ J. J. Walsh ${ }^{57 a}$ D. Lopes Pegna, ${ }^{58}$ J. Olsen, ${ }^{58}$ A. J. S. Smith, ${ }^{58}$ A. V. Telnov, ${ }^{58}$ F. Anulli, ${ }^{59 a}$ R. Faccini,,${ }^{59,59 b}$ F. Ferrarotto, ${ }^{59 a}$ F. Ferroni, ${ }^{59 a, 59 b}$ M. Gaspero, ${ }^{59 a, 59 b}$ L. Li Gioi, ${ }^{59 a}$ M. A. Mazzoni, ${ }^{59 a}$ G. Piredda, ${ }^{59 a}$ C. Bünger, ${ }^{60}$ O. Grünberg, ${ }^{60}$ T. Hartmann, ${ }^{60}$ T. Leddig,${ }^{60}$ H. Schröder,${ }^{60, *}$ C. Voss,${ }^{60}$ R. Waldi, ${ }^{60}$ T. Adye, ${ }^{61}$ E. O. Olaiya,${ }^{61}$ F. F. Wilson, ${ }^{61}$ S. Emery, ${ }^{62}$ G. Hamel de Monchenault, ${ }^{62}$ G. Vasseur, ${ }^{62}$ Ch. Yèche, ${ }^{62}$ D. Aston, ${ }^{63}$ D. J. Bard, ${ }^{63}$

R. Bartoldus ${ }^{63}$ J.F. Benitez ${ }^{63}$ C. Cartaro, ${ }^{63}$ M. R. Convery ${ }^{63}$ J. Dorfan, ${ }^{63}$ G. P. Dubois-Felsmann, ${ }^{63}$ W. Dunwoodie,${ }^{63}$ M. Ebert, ${ }^{63}$ R. C. Field ${ }^{63}$ M. Franco Sevilla, ${ }^{63}$ B. G. Fulsom, ${ }^{63}$ A. M. Gabareen, ${ }^{63}$ M. T. Graham, ${ }^{63}$ P. Grenier, ${ }^{63}$ C. Hast, ${ }^{63}$ W. R. Innes, ${ }^{63}$ M. H. Kelsey, ${ }^{63}$ P. Kim, ${ }^{63}$ M. L. Kocian, ${ }^{63}$ D. W. G. S. Leith,${ }^{63}$ P. Lewis, ${ }^{63}$ B. Lindquist, ${ }^{63}$ S. Luitz, ${ }^{63}$ V. Luth, ${ }^{63}$ H. L. Lynch, ${ }^{63}$ D. B. MacFarlane, ${ }^{63}$ D. R. Muller ${ }^{63}$ H. Neal, ${ }^{63}$ S. Nelson, ${ }^{63}$ M. Perl, ${ }^{63}$ T. Pulliam, ${ }^{63}$ B. N. Ratcliff, ${ }^{63}$ A. Roodman, ${ }^{63}$ A. A. Salnikov, ${ }^{63}$ R. H. Schindler, ${ }^{63}$ A. Snyder, ${ }^{63}$ D. Su,${ }^{63}$ M. K. Sullivan, ${ }^{63}$ J. Va'vra, ${ }^{63}$

A. P. Wagner, ${ }^{63}$ W. J. Wisniewski, ${ }^{63}$ M. Wittgen ${ }^{63}$ D. H. Wright, ${ }^{63}$ H. W. Wulsin,${ }^{63}$ C. C. Young, ${ }^{63}$ V. Ziegler, ${ }^{63}$

W. Park, ${ }^{64}$ M. V. Purohit, ${ }^{64}$ R. M. White, ${ }^{64}$ J.R. Wilson, ${ }^{64}$ A. Randle-Conde, ${ }^{65}$ S. J. Sekula, ${ }^{65}$ M. Bellis, ${ }^{66}$

P. R. Burchat, ${ }^{66}$ T. S. Miyashita, ${ }^{66}$ M. S. Alam, ${ }^{67}$ J. A. Ernst, ${ }^{67}$ R. Gorodeisky, ${ }^{68}$ N. Guttman, ${ }^{68}$ D. R. Peimer, ${ }^{68}$ A. Soffer, ${ }^{68}$ P. Lund, ${ }^{69}$ S. M. Spanier, ${ }^{69}$ J. L. Ritchie, ${ }^{70}$ A. M. Ruland, ${ }^{70}$ R. F. Schwitters, ${ }^{70}$ B. C. Wray, ${ }^{70}$ J. M. Izen, ${ }^{71}$ X. C. Lou, ${ }^{71}$ F. Bianchi, ${ }^{72 a, 72 b}$ D. Gamba, ${ }^{72 a, 72 b}$ S. Zambito, ${ }^{72 a, 72 b}$ L. Lanceri, ${ }^{73 a, 73 b}$ L. Vitale, ${ }^{73 a, 73 b}$ F. Martinez-Vidal, ${ }^{74}$

A. Oyanguren, ${ }^{74}$ H. Ahmed, ${ }^{75}$ J. Albert, ${ }^{75}$ Sw. Banerjee, ${ }^{75}$ F. U. Bernlochner, ${ }^{75}$ H. H. F. Choi, ${ }^{75}$ G. J. King,${ }^{75}$ R. Kowalewski, ${ }^{75}$ M. J. Lewczuk, ${ }^{75}$ I. M. Nugent, ${ }^{75}$ J. M. Roney, ${ }^{75}$ R. J. Sobie, ${ }^{75}$ N. Tasneem, ${ }^{75}$ T. J. Gershon, ${ }^{76}$ P. F. Harrison, ${ }^{76}$ T. E. Latham,${ }^{76}$ E. M. T. Puccio, ${ }^{76}$ H. R. Band, ${ }^{77}$ S. Dasu,${ }^{77}$ Y. Pan, ${ }^{77}$ R. Prepost,${ }^{77}$ and S. L. Wu ${ }^{77}$ 
(BABAR Collaboration)

\author{
${ }^{1}$ Laboratoire d'Annecy-le-Vieux de Physique des Particules (LAPP), Université de Savoie, CNRS/IN2P3, \\ F-74941 Annecy-Le-Vieux, France \\ ${ }^{2}$ Universitat de Barcelona, Facultat de Fisica, Departament ECM, E-08028 Barcelona, Spain \\ ${ }^{3 a}$ INFN Sezione di Bari, I-70126 Bari, Italy \\ ${ }^{3 \mathrm{~b}}$ Dipartimento di Fisica, Università di Bari, I-70126 Bari, Italy \\ ${ }^{4}$ University of Bergen, Institute of Physics, N-5007 Bergen, Norway \\ ${ }^{5}$ Lawrence Berkeley National Laboratory and University of California, Berkeley, California 94720, USA \\ ${ }^{6}$ Ruhr Universität Bochum, Institut für Experimentalphysik 1, D-44780 Bochum, Germany \\ ${ }^{7}$ University of British Columbia, Vancouver, British Columbia, Canada V6T 1 Z1 \\ ${ }^{8}$ Brunel University, Uxbridge, Middlesex UB8 3PH, United Kingdom \\ ${ }^{9}$ Budker Institute of Nuclear Physics, Novosibirsk 630090, Russia \\ ${ }^{10}$ University of California at Irvine, Irvine, California 92697, USA \\ ${ }^{11}$ University of California at Riverside, Riverside, California 92521, USA \\ ${ }^{12}$ University of California at Santa Barbara, Santa Barbara, California 93106, USA \\ ${ }^{13}$ University of California at Santa Cruz, Institute for Particle Physics, Santa Cruz, California 95064, USA \\ ${ }^{14}$ California Institute of Technology, Pasadena, California 91125, USA \\ ${ }^{15}$ University of Cincinnati, Cincinnati, Ohio 45221, USA \\ ${ }^{16}$ University of Colorado, Boulder, Colorado 80309, USA \\ ${ }^{17}$ Colorado State University, Fort Collins, Colorado 80523, USA \\ ${ }^{18}$ Technische Universität Dortmund, Fakultät Physik, D-44221 Dortmund, Germany \\ ${ }^{19}$ Technische Universität Dresden, Institut für Kern- und Teilchenphysik, D-01062 Dresden, Germany \\ ${ }^{20}$ Laboratoire Leprince-Ringuet, Ecole Polytechnique, CNRS/IN2P3, F-91128 Palaiseau, France \\ ${ }^{21}$ University of Edinburgh, Edinburgh EH9 3JZ, United Kingdom \\ ${ }^{22 \mathrm{a}}$ INFN Sezione di Ferrara, I-44100 Ferrara, Italy \\ ${ }^{22 \mathrm{~b}}$ Dipartimento di Fisica, Università di Ferrara, I-44100 Ferrara, Italy \\ ${ }^{23}$ INFN Laboratori Nazionali di Frascati, I-00044 Frascati, Italy \\ ${ }^{24 a}$ INFN Sezione di Genova, I-16146 Genova, Italy \\ ${ }^{24 \mathrm{~b}}$ Dipartimento di Fisica, Università di Genova, I-16146 Genova, Italy \\ ${ }^{25}$ Indian Institute of Technology Guwahati, Guwahati, Assam, 781 039, India \\ ${ }^{26}$ Harvard University, Cambridge, Massachusetts 02138, USA \\ ${ }^{27}$ Harvey Mudd College, Claremont, California 91711, USA \\ ${ }^{28}$ Universität Heidelberg, Physikalisches Institut, Philosophenweg 12, D-69120 Heidelberg, Germany \\ ${ }^{29}$ Humboldt-Universität zu Berlin, Institut für Physik, Newtonstrasse 15, D-12489 Berlin, Germany \\ ${ }^{30}$ Imperial College London, London, SW7 2AZ, United Kingdom \\ ${ }^{31}$ University of Iowa, Iowa City, Iowa 52242, USA \\ ${ }^{32}$ Iowa State University, Ames, Iowa 50011-3160, USA \\ ${ }^{33}$ Johns Hopkins University, Baltimore, Maryland 21218, USA \\ ${ }^{34}$ Laboratoire de l'Accélérateur Linéaire, IN2P3/CNRS et Université Paris, Sud 11, Centre de Scientifique d'Orsay, \\ B. P. 34, F-91898 Orsay Cedex, France \\ ${ }^{35}$ Lawrence Livermore National Laboratory, Livermore, California 94550, USA \\ ${ }^{36}$ University of Liverpool, Liverpool L69 7ZE, United Kingdom \\ ${ }^{37}$ Queen Mary, University of London, London, E1 4NS, United Kingdom \\ ${ }^{38}$ University of London, Royal Holloway and Bedford New College, Egham, Surrey TW20 OEX, United Kingdom \\ ${ }^{39}$ University of Louisville, Louisville, Kentucky 40292, USA \\ ${ }^{40}$ Johannes Gutenberg-Universität Mainz, Institut für Kernphysik, D-55099 Mainz, Germany \\ ${ }^{41}$ University of Manchester, Manchester M13 9PL, United Kingdom \\ ${ }^{42}$ University of Maryland, College Park, Maryland 20742, USA \\ ${ }^{43}$ University of Massachusetts, Amherst, Massachusetts 01003, USA \\ ${ }^{44}$ Massachusetts Institute of Technology, Laboratory for Nuclear Science, Cambridge, Massachusetts 02139, USA \\ ${ }^{45}$ McGill University, Montréal, Québec, Canada H3A $2 T 8$ \\ ${ }^{46 a}$ INFN Sezione di Milano, I-20133 Milano, Italy \\ ${ }^{46 \mathrm{~b}}$ Dipartimento di Fisica, Università di Milano, I-20133 Milano, Italy \\ ${ }^{47}$ University of Mississippi, University, Mississippi 38677, USA \\ ${ }^{48}$ Université de Montréal, Physique des Particules, Montréal, Québec, Canada H3C 3J7 \\ ${ }^{49}$ INFN Sezione di Napoli, I-80126 Napoli, Italy \\ ${ }^{49 \mathrm{~b}}$ Dipartimento di Scienze Fisiche, Università di Napoli Federico II, I-80126 Napoli, Italy \\ ${ }^{50}$ NIKHEF, National Institute for Nuclear Physics and High Energy Physics, NL-1009 DB Amsterdam, The Netherlands
}


${ }^{51}$ University of Notre Dame, Notre Dame, Indiana 46556, USA

${ }^{52}$ Ohio State University, Columbus, Ohio 43210, USA

${ }^{53}$ University of Oregon, Eugene, Oregon 97403, USA

${ }^{54 a}$ INFN Sezione di Padova, I-35131 Padova, Italy

${ }^{54 b}$ Dipartimento di Fisica, Università di Padova, I-35131 Padova, Italy

${ }^{55}$ Laboratoire de Physique Nucléaire et de Hautes Energies, IN2P3/CNRS, Université Pierre et Marie Curie-Paris6,

Université Denis Diderot-Paris7, F-75252 Paris, France

${ }^{56}$ INFN Sezione di Perugia, I-06100 Perugia, Italy

${ }^{56 \mathrm{~b}}$ Dipartimento di Fisica, Università di Perugia, I-06100 Perugia, Italy

${ }^{57}$ INFN Sezione di Pisa, I-56127 Pisa, Italy

${ }^{57 \mathrm{~b}}$ Dipartimento di Fisica, Università di Pisa, I-56127 Pisa, Italy

${ }^{57 \mathrm{c}}$ Scuola Normale Superiore di Pisa, I-56127 Pisa, Italy

${ }^{58}$ Princeton University, Princeton, New Jersey 08544, USA

${ }^{59 a}$ INFN Sezione di Roma, I-00185 Roma, Italy

${ }^{59 \mathrm{~b}}$ Dipartimento di Fisica, Università di Roma La Sapienza, I-00185 Roma, Italy

${ }^{60}$ Universität Rostock, D-18051 Rostock, Germany

${ }^{61}$ Rutherford Appleton Laboratory, Chilton, Didcot, Oxon OX11 0QX, United Kingdom

${ }^{62}$ CEA, Irfu, SPP, Centre de Saclay, F-91191 Gif-sur-Yvette, France

${ }^{63}$ SLAC National Accelerator Laboratory, Stanford, California 94309 USA

${ }^{64}$ University of South Carolina, Columbia, South Carolina 29208, USA

${ }^{65}$ Southern Methodist University, Dallas, Texas 75275, USA

${ }^{66}$ Stanford University, Stanford, California 94305-4060, USA

${ }^{67}$ State University of New York, Albany, New York 12222, USA

${ }^{68}$ Tel Aviv University, School of Physics and Astronomy, Tel Aviv 69978, Israel

${ }^{69}$ University of Tennessee, Knoxville, Tennessee 37996, USA

${ }^{70}$ University of Texas at Austin, Austin, Texas 78712, USA

${ }^{71}$ University of Texas at Dallas, Richardson, Texas 75083, USA

${ }^{72 \mathrm{a}}$ INFN Sezione di Torino, I-10125 Torino, Italy

${ }^{72 b}$ Dipartimento di Fisica Sperimentale, Università di Torino, I-10125 Torino, Italy

${ }^{73 a}$ INFN Sezione di Trieste, I-34127 Trieste, Italy

${ }^{73 b}$ Dipartimento di Fisica, Università di Trieste, I-34127 Trieste, Italy

${ }^{74}$ IFIC, Universitat de Valencia-CSIC, E-46071 Valencia, Spain

${ }^{75}$ University of Victoria, Victoria, British Columbia, Canada V8W $3 P 6$

${ }^{76}$ Department of Physics, University of Warwick, Coventry CV4 7AL, United Kingdom

${ }^{77}$ University of Wisconsin, Madison, Wisconsin 53706, USA

(Received 8 July 2012; published 8 August 2013)

We present a search for the decay $B^{+} \rightarrow \tau^{+} \nu$ using $467.8 \times 10^{6} B \bar{B}$ pairs collected at the $Y(4 S)$ resonance with the $B A B A R$ detector at the SLAC PEP-II $B$-Factory. We select a sample of events with one completely reconstructed $B^{-}$in the hadronic decay mode $\left(B^{-} \rightarrow D^{(*) 0} X^{-}\right.$and $\left.B^{-} \rightarrow J / \psi X^{-}\right)$. We examine the rest of the event to search for a $B^{+} \rightarrow \tau^{+} \nu$ decay. We identify the $\tau^{+}$lepton in the following modes: $\tau^{+} \rightarrow e^{+} \nu \bar{\nu}, \tau^{+} \rightarrow \mu^{+} \nu \bar{\nu}, \tau^{+} \rightarrow \pi^{+} \bar{\nu}$ and $\tau^{+} \rightarrow \rho^{+} \bar{\nu}$. We find an excess of events with respect to the expected background, which excludes the null signal hypothesis at the level of $3.8 \sigma$ (including systematic uncertainties) and corresponds to a branching fraction value of $\mathcal{B}\left(B^{+} \rightarrow \tau^{+} \nu\right)=$ $\left(1.83_{-0.49}^{+0.53}(\right.$ stat $) \pm 0.24($ syst $\left.)\right) \times 10^{-4}$.

DOI: 10.1103/PhysRevD.88.031102

PACS numbers: $13.20 .-\mathrm{v}, 13.25 . \mathrm{Hw}$

\footnotetext{
*Deceased.

Present address: University of Tabuk, Tabuk 71491, Saudi Arabia.

${ }^{\ddagger}$ Also at Università di Perugia, Dipartimento di Fisica, Perugia, Italy.

${ }^{\S}$ Present address: University of Huddersfield, Huddersfield HD1 3DH, United Kingdom.

"Present address: University of South Alabama, Mobile, Alabama 36688, USA.

${ }_{\text {II }}$ Also at Università di Sassari, Sassari, Italy.
}

The study of the purely leptonic decay $B^{+} \rightarrow \tau^{+} \nu[1]$ is of particular interest to test the predictions of the Standard Model (SM) and to probe new physics effects. It is sensitive to the product of the $B$ meson decay constant $f_{B}$, and the absolute value of the Cabibbo-Kobayashi-Maskawa matrix element $\left|V_{u b}\right|$ [2]. In the SM the branching fraction is given by

$\mathcal{B}\left(B^{+} \rightarrow \tau^{+} \nu\right)=\frac{G_{F}^{2} m_{B} m_{\tau}^{2}}{8 \pi}\left[1-\frac{m_{\tau}^{2}}{m_{B}^{2}}\right]^{2} f_{B}^{2}\left|V_{u b}\right|^{2} \tau_{B^{+}}$,

where $G_{F}$ is the Fermi constant, $m_{B}$ and $m_{\tau}$ are the $B^{+}$meson and $\tau$ lepton masses, respectively, and $\tau_{B^{+}}$is the $B^{+}$lifetime. 
Using the lattice QCD calculation of $f_{B}=(189 \pm 4) \mathrm{MeV}$ [3], and the BABAR measurement of $\left|V_{u b}\right|$ from charmless semileptonic $B$ exclusive decays [4], the predicted SM value of the brancing fraction is $\mathcal{B}_{\mathrm{SM}}\left(B^{+} \rightarrow \tau^{+} \nu\right)=(0.62 \pm$ $0.12) \times 10^{-4}$. If we use the $B A B A R$ measurement of $\left|V_{u b}\right|$ from inclusive charmless semileptonic B decays [5], the SM prediction is $\mathcal{B}_{\mathrm{SM}}\left(B^{+} \rightarrow \tau^{+} \nu\right)=(1.18 \pm 0.16) \times 10^{-4}$.

The process is sensitive to possible extensions of the SM. For instance, in two-Higgs doublet models (2HDM) [6] and in minimal supersymmetric extensions [7], it can be mediated by a charged Higgs boson. A branching fraction measurement can, therefore, also be used to constrain the parameter space of new physics models.

The data used in this analysis were collected with the $B A B A R$ detector at the PEP-II storage ring. The sample corresponds to an integrated luminosity of $426 \mathrm{fb}^{-1}$ at the $\Upsilon(4 S)$ resonance. The sample contains $(467.8 \pm 5.1) \times$ $10^{6} B \bar{B}$ decays $\left(N_{B \bar{B}}\right)$. The detector is described in detail elsewhere [8]. Charged particle trajectories are measured in the tracking system composed of a five-layer doublesided silicon vertex tracker and a 40-layer drift chamber, operating in a $1.5 \mathrm{~T}$ solenoidal magnetic field. A Cherenkov detector is used for charged $\pi-K$ discrimination, a CsI calorimeter for photon and electron identification, and the flux return of the solenoid, which consists of layers of iron interspersed with resistive plate chambers or limited streamer tubes, for muon and neutral hadron identification.

We use a Monte Carlo (MC) simulation based on GEANT4 [9] to estimate signal selection efficiencies and to study backgrounds. In MC simulated signal events, one $B^{+}$meson decays as $B^{+} \rightarrow \tau^{+} \nu$ and the other decays in any final state. The $B \bar{B}$ and continuum MC samples are equivalent to approximately 3 times and 1.5 times the data sample, respectively. Beam-related background and detector noise are sampled from data and overlaid on the simulated events.

We reconstruct an exclusive decay of one of the $B$ mesons in the event (which we refer to as the tag- $B$ ) and examine the rest of the event for the experimental signature of $B^{+} \rightarrow \tau^{+} \nu$. The tag- $B$ reconstruction can be performed by looking at both hadronic $B$ decays and semileptonic $B$ decays. Published results from both BABAR and Belle are summarized in Table I.

We reconstruct the tag- $B$ candidate in the set of hadronic decays $B^{-} \rightarrow M^{0} X^{-}$, where $M^{0}$ denotes a $D^{(*) 0}$ or a $J / \psi$, and $X^{-}$denotes a system of hadrons with total charge -1 composed of $n_{1} \pi^{ \pm}, n_{2} K^{ \pm}, n_{3} \pi^{0}, n_{4} K_{S}^{0}$ where $n_{1}+n_{2} \leq$ $5, n_{2}, n_{3}$ and $n_{4} \leq 2$. We reconstruct the $D^{0}$ as $D^{0} \rightarrow$ $K^{-} \pi^{+}, \quad K^{-} \pi^{+} \pi^{0}, \quad K^{-} \pi^{+} \pi^{-} \pi^{+}, \quad K_{S}^{0} \pi^{0}, \quad K_{S}^{0} \pi^{+} \pi^{-}$, $K_{S}^{0} \pi^{+} \pi^{-} \pi^{0}, K^{+} K^{-}$, or $\pi^{+} \pi^{-}$. We reconstruct the $D^{* 0}$ meson as $D^{* 0} \rightarrow D^{0} \pi^{0}, D^{0} \gamma$, and the $J / \psi$ meson via their decays $J / \psi \rightarrow e^{+} e^{-}, \mu^{+} \mu^{-}$. Two kinematic variables are used to discriminate between correctly reconstructed tag- $B$ candidates and misreconstructed events: the beam
TABLE I. Published results for $B^{+} \rightarrow \tau^{+} \nu$ from $B A B A R$ and Belle collaborations.

\begin{tabular}{lcc}
\hline \hline Experiment & Tag & Branching fraction $\left(\times 10^{-4}\right)$ \\
\hline$B A B A R$ & Hadronic [10] & $1.8_{-0.8}^{+0.9} \pm 0.4 \pm 0.2$ \\
$B A B A R$ & Semileptonic [11] & $1.7 \pm 0.8 \pm 0.2$ \\
Belle & Hadronic [12] & $0.72_{-0.25}^{+0.27} \pm 0.11$ \\
Belle & Semileptonic [13] & $1.54_{-0.37-0.31}^{+0.38+0.29}$ \\
\hline \hline
\end{tabular}

energy-substituted mass $m_{\mathrm{ES}} \equiv \sqrt{s / 4-p_{B}^{2}}$, and the energy difference $\Delta \mathrm{E} \equiv E_{B}-\sqrt{s} / 2$, where $\sqrt{s}$ is the total energy in the $Y(4 S)$ center-of-mass $(\mathrm{CM})$ system and $p_{B}$ and $E_{B}$ respectively denote the momentum and the energy of the tag- $B$ candidate in the CM. The resolution on $\Delta E$ is measured to be $\sigma_{\Delta E}=10-35 \mathrm{MeV}$, depending on the decay mode; we require $|\Delta E|<3 \sigma_{\Delta E}$. Events with a $\operatorname{tag}-B$ candidate arise from two possible classes with different $m_{\mathrm{ES}}$ distributions. One class includes signal events with a correctly reconstructed tag- $B$, and background events from $Y(4 S) \rightarrow B^{+} B^{-}$with a correctly reconstructed tag- $B$. All these events are characterized by an $m_{\mathrm{ES}}$ distribution peaked at the nominal $B$ mass (signal and peaking background). The other classes of events consist of continuum background, $e^{+} e^{-} \rightarrow q \bar{q}(q=u, d, s, c)$ and $e^{+} e^{-} \rightarrow \tau^{+} \tau^{-}$, and combinatorial background, $\Upsilon(4 S) \rightarrow B^{0} \bar{B}^{0}$ or $B^{+} B^{-}$in which the tag- $B$ is misreconstructed. These events are characterized by a smooth $m_{\mathrm{ES}}$ distribution.

If multiple tag- $B$ candidates are reconstructed in the event, we select the one with the lowest value of $|\Delta E|$. After the reconstruction of the $\operatorname{tag}-B$, we require the presence of only one well-reconstructed track (signal track), with charge opposite to that of the tag- $B$. The purity $\mathcal{P}$ of each reconstructed tag- $B$ decay mode is estimated as the ratio of the number of peaking events with $m_{\mathrm{ES}}>$ $5.27 \mathrm{GeV}$ to the total number of events in the same range. The yield in data is determined by means of an extended unbinned maximum likelihood fit to the $m_{\mathrm{ES}}$ distribution, as shown in Fig. 1. We use a phenomenologically motivated threshold function (ARGUS function [14]) as probability density function (PDF) to describe the continuum and combinatorial background components in the fit, while for the correctly reconstructed tag- $B$ component we use a Gaussian distribution plus an exponential tail for the PDF (Crystal Ball function) [15]. We use only events with the tag- $B$ reconstructed in decay modes with $\mathcal{P}>0.1$. Combinatorial and continuum background distributions in any discriminating variable are estimated from a sideband in $m_{\mathrm{ES}}\left(5.209 \mathrm{GeV}<m_{\mathrm{ES}}<5.260 \mathrm{GeV}\right)$ and are extrapolated into the signal region $\left(m_{\mathrm{ES}}>5.270 \mathrm{GeV}\right)$ using the results of a fit to an ARGUS function. The peaking $B^{+} B^{-}$background shape is determined from $B^{+} B^{-}$MC, after subtraction of the combinatorial 


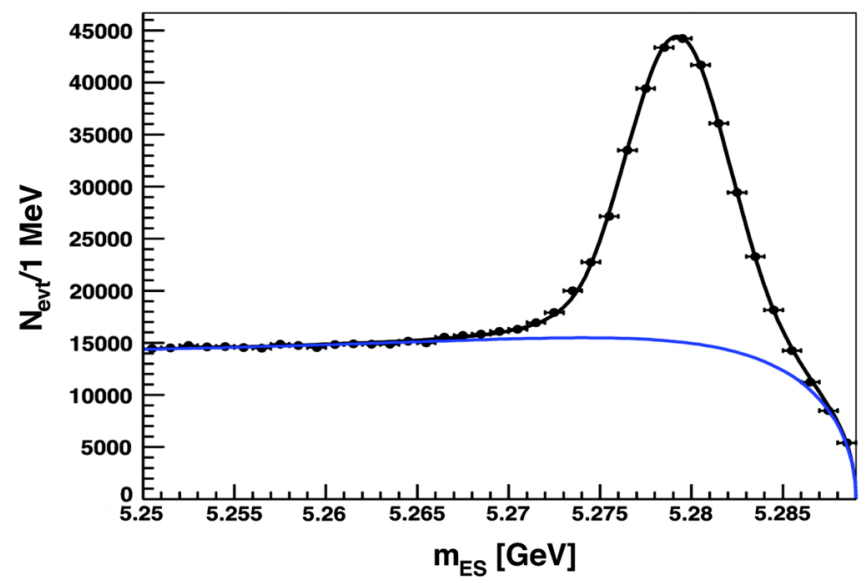

FIG. 1 (color online). Fit to the $m_{\mathrm{ES}}$ distribution in data. Dots are data, the upper curve is the global fit result and the lower curve represents the fitted combinatorial and continuum background.

component to avoid double counting. The efficiency of the tag- $B$ reconstruction in presence of a $B^{+} \rightarrow \tau^{+} \nu$ decay is estimated with signal MC as $\epsilon_{\mathrm{tag}}=(2.8 \pm 0.1) \times 10^{-3}$.

The signal-side $\tau$ lepton is reconstructed in four decay modes: $\tau^{+} \rightarrow e^{+} \nu \bar{\nu}, \quad \tau^{+} \rightarrow \mu^{+} \nu \bar{\nu}, \quad \tau^{+} \rightarrow \pi^{+} \nu$, and $\tau^{+} \rightarrow \rho^{+} \nu$, totaling approximately $70 \%$ of all $\tau$ decays. We separate the event sample into four categories using particle identification criteria applied to the signal track $\left(e^{+}, \mu^{+}\right.$, and $\left.\pi^{+}\right)$. The $\tau^{+} \rightarrow \rho^{+} \nu$ sample is obtained by associating the signal track $\pi^{+}$with a $\pi^{0}$ reconstructed from a pair of neutral clusters with an invariant mass between $115 \mathrm{MeV} / c^{2}$ and $155 \mathrm{MeV} / c^{2}$.

In order to remove the $e^{+} e^{-} \rightarrow \tau^{+} \tau^{-}$background, we impose $\tau$ mode dependent requirements on the ratio between the 2nd and the 0th Fox-Wolfram moments R2 [16] calculated using all the tracks and neutral clusters of the event. This preserves $90 \%$ of the $B^{+} \rightarrow \tau^{+} \nu$ signal.

To reject continuum background, we use the absolute value of $\cos \theta_{\mathrm{TB}}$, the cosine of the angle in the CM frame between the thrust axis [17] of the tag- $B$ and the thrust axis of the remaining charged and neutral candidates in the event. For correctly reconstructed tag- $B$ candidates the $\left|\cos \theta_{\mathrm{TB}}\right|$ distribution is expected to be uniform, while for jet-like $e^{+} e^{-} \rightarrow q \bar{q}$ continuum events it peaks strongly at 1 . In order to reject background from events with a correctly reconstructed tag- $B$, we study the distribution of several discriminating variables exploiting the different kinematics between the signal and background of the remaining reconstructed candidates. We use the missing momentum polar angle in the laboratory frame $\vec{p}_{\text {miss }}=$ $\vec{p}_{\mathrm{CM}}-\vec{p}_{\mathrm{tag} B}-\vec{p}_{\text {trk }}-\sum_{\text {neut }} \vec{p}_{i}$, where $\vec{p}_{\mathrm{CM}}$ is the total momentum of the beams, $\vec{p}_{\operatorname{tag} B}$ is the reconstructed momentum of the tag- $B$, and $\vec{p}_{\text {trk }}$ is the reconstructed track momentum, and the sum is extended on all the neutral candidates reconstructed in the calorimeter not assigned to the tag- $B$. For the $\tau^{+} \rightarrow \pi^{+} \nu$ mode, we combine $p_{\text {trk }}^{*}$ (where the star denotes the CM frame) and the cosine of the angle between $\vec{p}_{\text {miss }}$ and the beam axis $\left(\cos \theta_{\text {miss }}\right)$ in a likelihood ratio

$$
L_{P}=\frac{L_{S}\left(p_{\mathrm{trk}}^{*}, \cos \theta_{\mathrm{miss}}\right)}{\left(L_{S}\left(p_{\mathrm{trk}}^{*}, \cos \theta_{\mathrm{miss}}\right)+L_{B}\left(p_{\mathrm{trk}}^{*}, \cos \theta_{\mathrm{miss}}\right)\right)},
$$

where the signal (S) and background (B) likelihoods have been obtained from the product of the PDFs of the two discriminating variables: $L_{S}\left(p_{\text {trk }}^{*}, \cos \theta_{\text {miss }}\right)=$ $P_{S}\left(p_{\text {trk }}^{*}\right) P_{S}\left(\cos \theta_{\text {miss }}\right)$ and $L_{B}\left(p_{\text {trk }}^{*}, \cos \theta_{\text {miss }}\right)=P_{B}\left(p_{\text {trk }}^{*}\right) \times$ $P_{B}\left(\cos \theta_{\text {miss }}\right)$. Similarly, for the $\tau^{+} \rightarrow \rho^{+} \nu$ mode we combine four discriminating variables in the likelihood ratio $L_{P}: \cos \theta_{\text {miss }}$, the invariant mass of the $\pi^{0}$ candidate, the $\rho^{+}$candidate momentum, and the invariant mass of the $\pi^{+} \pi^{0}$ pair used to make the $\rho^{+}$candidate. The PDFs used in the likelihood ratio for the signal and background are determined from signal and $B^{+} B^{-}$MC samples, respectively.

The most powerful discriminating variable is $E_{\text {extra }}$, defined as the sum of the energies of the neutral clusters not associated with the tag- $B$ or with the signal $\pi^{0}$ from the $\tau^{+} \rightarrow \rho^{+} \nu$ mode, and passing a minimum energy requirement $(60 \mathrm{MeV})$. Signal events tend to peak at low $E_{\text {extra }}$. Background events, which contain additional sources of neutral clusters, tend to be distributed at higher values. The signal region in data is kept blind until the end of the analysis chain when we extract the signal yield, meaning that we do not use events in data with $E_{\text {extra }}<400 \mathrm{MeV}$ during the selection optimization procedure and for the evaluation of background shapes.

We optimize the selection requirements, including those on the purity $\mathcal{P}$ of the tag- $B$ and the minimum energy of the neutral clusters, minimizing the expected uncertainty in the branching fraction fit. In order to estimate the uncertainty, which includes the statistical and the dominant systematic sources, we run $1000 \mathrm{MC}$ simulated pseudo experiments extracted from the background and signal expected $E_{\text {extra }}$ distributions for a set of possible selection requirements, assuming a signal branching fraction of $1.8 \times 10^{-4}$ [10].

Table II summarizes the signal selection requirements and Fig. 2 shows the $E_{\text {extra }}$ distribution with all the selection requirements applied. The background events populating the low $E_{\text {extra }}$ region are mostly semileptonic $B$ decays for the leptonic modes. For the $\tau^{+} \rightarrow \pi^{+} \nu$ mode the background is composed mostly of charmless hadronic $B$

TABLE II. Optimized signal selection criteria for each $\tau$ mode.

\begin{tabular}{lcccc}
\hline \hline Variable & $e^{+}$ & $\mu^{+}$ & $\pi^{+}$ & $\rho^{+}$ \\
\hline $\mathcal{P}$ & \multicolumn{4}{c}{$>10 \%$} \\
Cluster energy $(\mathrm{MeV})$ & \multicolumn{4}{c}{$>60$} \\
$\mathcal{R} 2$ & $<0.57$ & $<0.56$ & $<0.56$ & $<0.51$ \\
$\left|\cos \theta_{\mathrm{TB}}\right|$ & $<0.95$ & $<0.90$ & $<0.65$ & $<0.8$ \\
$L_{P}$ & & & $>0.30$ & $>0.45$ \\
\hline \hline
\end{tabular}



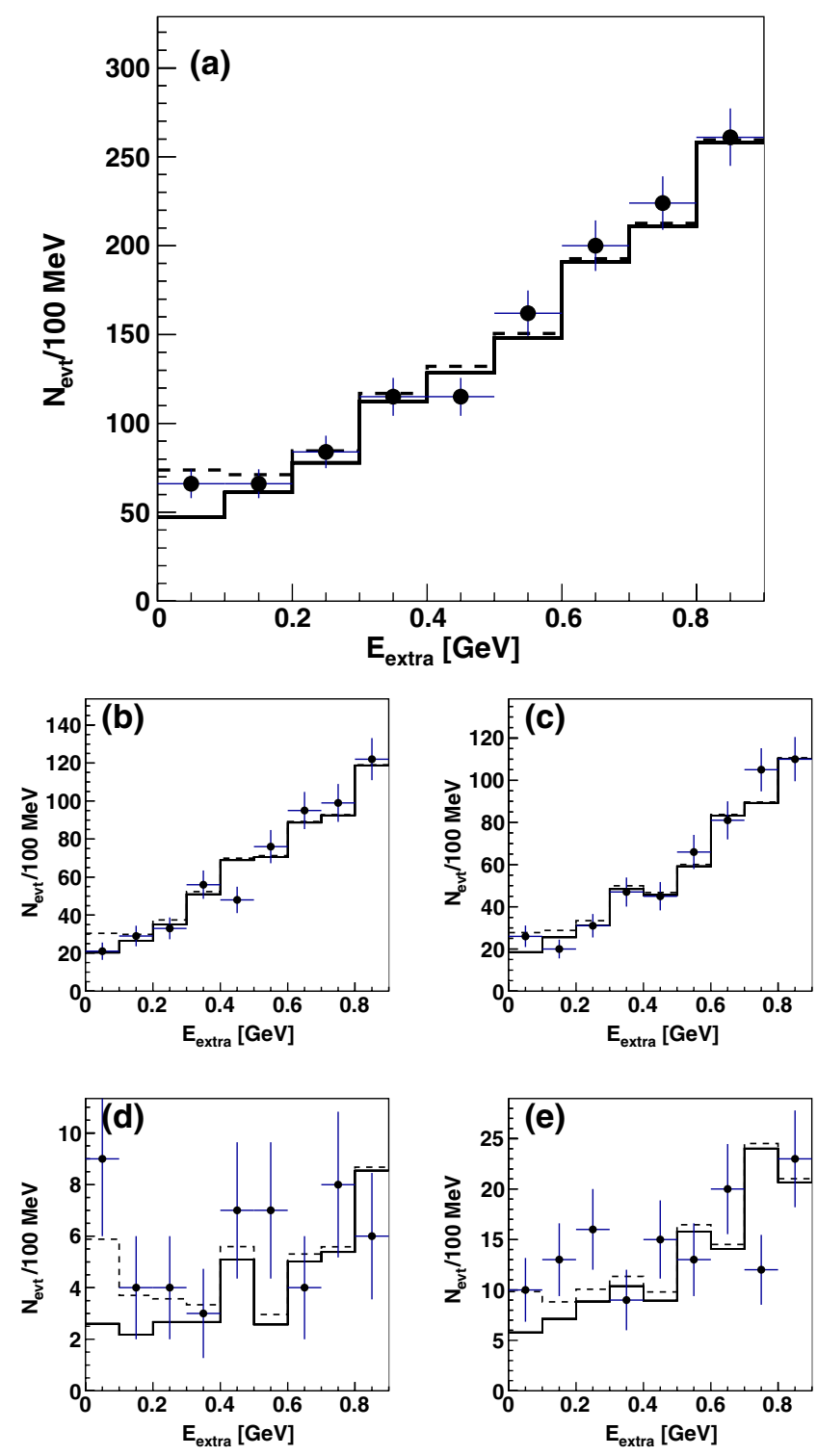

FIG. 2 (color online). $E_{\text {extra }}$ distribution in data (points with error bars) with all selection requirements applied and fit results overlaid. The hatched histogram is the background and the dashed component is the best-fit signal excess distribution. Plot (a) shows all $\tau$ decay modes fitted simultaneously. Lower plots show the projection of the simultaneous fit result on the four analyzed $\tau$ decay modes: (b) $\tau^{+} \rightarrow e^{+} \nu \bar{\nu}$, (c) $\tau^{+} \rightarrow \mu^{+} \nu \bar{\nu}$, (d) $\tau^{+} \rightarrow \pi^{+} \nu$, (e) $\tau^{+} \rightarrow \rho^{+} \nu$.

decays and semileptonic $B$ decays with a muon in the final state. For the $\tau^{+} \rightarrow \rho^{+} \nu$ mode the backgrounds are charmed hadronic $B$ decays, semileptonic $B$ decays with a muon in the final state and a small fraction with a $\tau$.

We use an extended unbinned maximum likelihood fit to the measured $E_{\text {extra }}$ distribution to extract the $B^{+} \rightarrow \tau^{+} \nu$ branching fraction. The likelihood function for the $N_{k}$ candidates selected in one of the four reconstructed $\tau$ decay modes $k$ is

$$
\mathcal{L}_{k}=\frac{e^{-\left(n_{s, k}+n_{b, k}\right)}}{N_{k} !} \prod_{i=1}^{N_{k}}\left\{n_{s, k} \mathcal{P}_{k}^{s}\left(E_{i, k}\right)+n_{b, k} \mathcal{P}_{k}^{b}\left(E_{i, k}\right)\right\}
$$

where $n_{s, k}$ is the signal yield, $n_{b, k}$ is the background yield, $E_{i, k}$ is the $E_{\text {extra }}$ value of the $i$ th event, $\mathcal{P}_{k}^{s}$ is the PDF of signal events, and $\mathcal{P}_{k}^{b}$ is the PDF of background events. The background yields in each decay mode are permitted to float independently of each other in the fit, while the signal yields are constrained to a single branching ratio via the relation

$$
n_{s, k}=N_{B \bar{B}} \times \epsilon_{k} \times \mathcal{B},
$$

where $\epsilon_{k}$ is the reconstruction efficiency of the signal $B^{+} \rightarrow \tau^{+} \nu$ decay in the $k$ reconstructed $\tau$ decay mode, and $\mathcal{B}$ is the $B^{+} \rightarrow \tau^{+} \nu$ branching fraction. The parameters $N_{B \bar{B}}$ and $\epsilon_{k}$ are fixed in the fit while $\mathcal{B}$ is allowed to vary. The reconstruction efficiencies $\epsilon_{k}$, which include signal cross-feeds among $\tau$ reconstruction modes and $\tau$ branching fractions, are obtained from MC-simulated signal events (see Table III). Since the tag- $B$ reconstruction efficiency is included in $\epsilon_{k}$ and is estimated from the signal $\mathrm{MC}$, we apply a correction factor of $R_{\mathrm{data} / \mathrm{MC}}=0.926 \pm$ 0.010 to take into account data/MC differences. This is derived from the ratio of the peaking component of the $m_{\mathrm{ES}}$ distribution for the hadronic tag- $B$ in data and in $\mathrm{MC}$ simulated events.

The signal PDF is an histogram obtained from a high statistics signal sample of MC simulated data. We use a sample of fully reconstructed events to correct the signal $\mathrm{PDF}$ for data/MC disagreement. In addition to the reconstructed tag- $B$, a second $B$ is reconstructed in the hadronic or the semileptonic decay mode using tracks and neutral clusters not assigned to the tag- $B$. In order to estimate the correction to the signal PDF, we compare the distribution of $E_{\text {extra }}$ in this double tagged event sample from experimental data and MC simulations. The MC distributions are normalized to the experimental data and the comparison is shown in Fig. 3. We extract the correction function by taking the ratio of the two distributions and fitting it with a second order polynomial.

TABLE III. Reconstruction efficiency $\epsilon_{k}$, measured branching fractions, and statistical uncertainty obtained from the fit with all the modes separately and constrained to the same branching fraction. The $\tau$ decay mode branching fractions are included in the efficiencies.

\begin{tabular}{lccc}
\hline \hline Decay mode & $\epsilon_{k}\left(\times 10^{-4}\right)$ & Signal yield & $\mathcal{B}\left(\times 10^{-4}\right)$ \\
\hline$\tau^{+} \rightarrow e^{+} \nu \bar{\nu}$ & $2.47 \pm 0.14$ & $4.1 \pm 9.1$ & $0.35_{-0.73}^{+0.84}$ \\
$\tau^{+} \rightarrow \mu^{+} \nu \bar{\nu}$ & $2.45 \pm 0.14$ & $12.9 \pm 9.7$ & $1.12_{-0.78}^{+0.90}$ \\
$\tau^{+} \rightarrow \pi^{+} \nu$ & $0.98 \pm 0.14$ & $17.1 \pm 6.2$ & $3.69_{-1.22}^{+1.42}$ \\
$\tau^{+} \rightarrow \rho^{+} \nu$ & $1.35 \pm 0.11$ & $24.0 \pm 10.0$ & $3.78_{-1.45}^{+1.65}$ \\
Combined & & $62.1 \pm 17.3$ & $1.83_{-0.49}^{+0.53}$ \\
\hline \hline
\end{tabular}



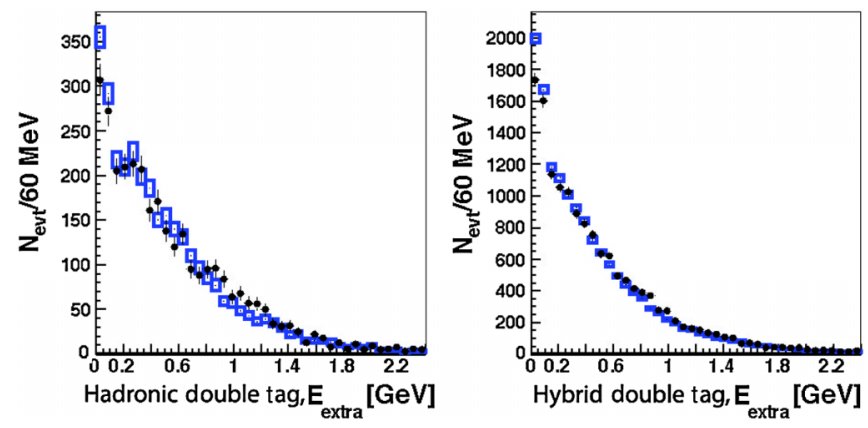

FIG. 3 (color online). $E_{\text {extra }}$ distribution for double tagged events. The "signal" $B$ is reconstructed in hadronic decays (left plot) or semileptonic decays (right plot). Points are data and boxes are MC simulation.

We determine the PDF of the combinatorial background from the $m_{\mathrm{ES}}$ sideband. The normalization of this component in the signal region is obtained by fitting the $m_{\mathrm{ES}}$ distribution after the selection has been applied. The shape of the peaking background is taken from $B^{+} B^{-} \mathrm{MC}$. The two background components are added together into a single histogram background PDF. We estimate the branching fraction by minimizing $-\ln \mathcal{L}$, where $\mathcal{L}=\Pi_{k=1}^{4} \mathcal{L}_{k}$, and $\mathcal{L}_{k}$ is given in Eq. (3). The projections of the fit results are shown in Fig. 2.

We observe an excess of events with respect to the expected background level and measure a branching fraction of $\mathcal{B}\left(B^{+} \rightarrow \tau^{+} \nu\right)=\left(1.83_{-0.49}^{+0.53}\right) \times 10^{-4}$, where the uncertainty is statistical. Table III summarizes the results from the fit. We evaluate the significance of the observed signal, including only statistical uncertainty, as $S=$ $\sqrt{2 \ln \left(\mathcal{L}_{s+b} / \mathcal{L}_{b}\right)}$, where $\mathcal{L}_{s+b}$ and $\mathcal{L}_{b}$ denote the obtained maximum likelihood values in the signal and background, and the background only hypotheses, respectively. We find $S=4.2 \sigma$.

Additive systematic uncertainties are due to the uncertainties in the signal and background $E_{\text {extra }}$ PDF shapes used in the fit. To estimate the systematic uncertainty in the background PDF shape we repeat the fit of the branching fraction with 1000 variations of the background PDFs, varying each bin content within its statistical uncertainty. We use the range of fitted branching fractions covering $68 \%$ of the distribution as systematic uncertainty yielding an overall contribution of $10 \%$. We correct the systematic effects of disagreements between data and MC $E_{\text {extra }}$ distributions for signal events using a sample of completely reconstructed events in data and MC, as already described. To estimate the related systematic uncertainties, we vary the parameters of the second-order polynomial defining the correction within their uncertainty and repeat the fit to the $B^{+} \rightarrow \tau^{+} \nu$ branching fraction. We observe a $2.6 \%$ variation that we take as the systematic uncertainty on the signal shape. Including the effects of additive systematic uncertainties, the significance of the result is evaluated as $3.8 \sigma$.
TABLE IV. Contributions to systematic uncertainty on the branching fraction.

\begin{tabular}{lc}
\hline \hline Source of systematics & $\mathcal{B}$ uncertainty $(\%)$ \\
\hline Additive & \\
Background PDF & 10 \\
Signal PDF & 2.6 \\
Multiplicative & \\
Tag- $B$ efficiency & 5.0 \\
$B$ counting & 1.1 \\
Electron identification & 2.6 \\
Muon identification & 4.7 \\
Kaon identification & 0.4 \\
Tracking & 0.5 \\
MC statistics & 0.6 \\
Total & 13 \\
\hline \hline
\end{tabular}

Multiplicative systematic uncertainties on the efficiency stem from the uncertainty in the tag- $B$ efficiency correction (5.0\%), estimated by comparing the ratio of double tags yield in data and in MC simulation with the same ratio for single tags, electron identification (2.6\%), muon identification $(4.7 \%)$, charged kaon veto $(0.4 \%)$, estimated from experimental data control samples, and the finite signal MC statistics (0.8\%). Table IV summarizes the systematic uncertainties. The total systematic uncertainty is obtained by combining all sources in quadrature.

In summary, we have measured the branching fraction of the decay $B^{+} \rightarrow \tau^{+} \nu$ using a tagging algorithm based on the reconstruction of hadronic $B$ decays using a data sample of $467.8 \times 10^{6} B \bar{B}$ pairs collected with the $B A B A R$ detector at the PEP-II $B$-Factory. We measure the branching fraction to be $\mathcal{B}\left(B^{+} \rightarrow \tau^{+} \nu\right)=\left(1.83_{-0.49}^{+0.53}\right.$ (stat) \pm 0.24 (syst) $) \times 10^{-4}$, excluding the null hypothesis by $3.8 \sigma$ (including systematic uncertainty). This result supersedes our previous result using the same technique [10]. The improvements in the statistical and systematic uncertainties are due to the cumulative effect of several factors, which we briefly list in the following, for the interested reader. We improved the tag-B reconstruction algorithm, considering more decay modes with the effect of increasing the efficiency by a factor 2 , at the cost of a larger background of misreconstructed tag- $B$ s. We performed a multivariate analysis choosing the variables and the selection level by an optimisation procedure aiming at the smallest uncertainty, by means of Monte Carlo pseudoexperiments. To extract the signal yield, the previous analysis used a cut and count method, while we fit the signal yield maximising a likelihood built from the most discriminating variable. Finally, the present analysis took advantage of a more recent version of the reconstruction software and data Monte Carlo studies to assess systematic uncertainties.

The result is statistically consistent with recent Belle measurement using a similar tag- $B$ reconstruction 


\section{J. P. LEES et al.}
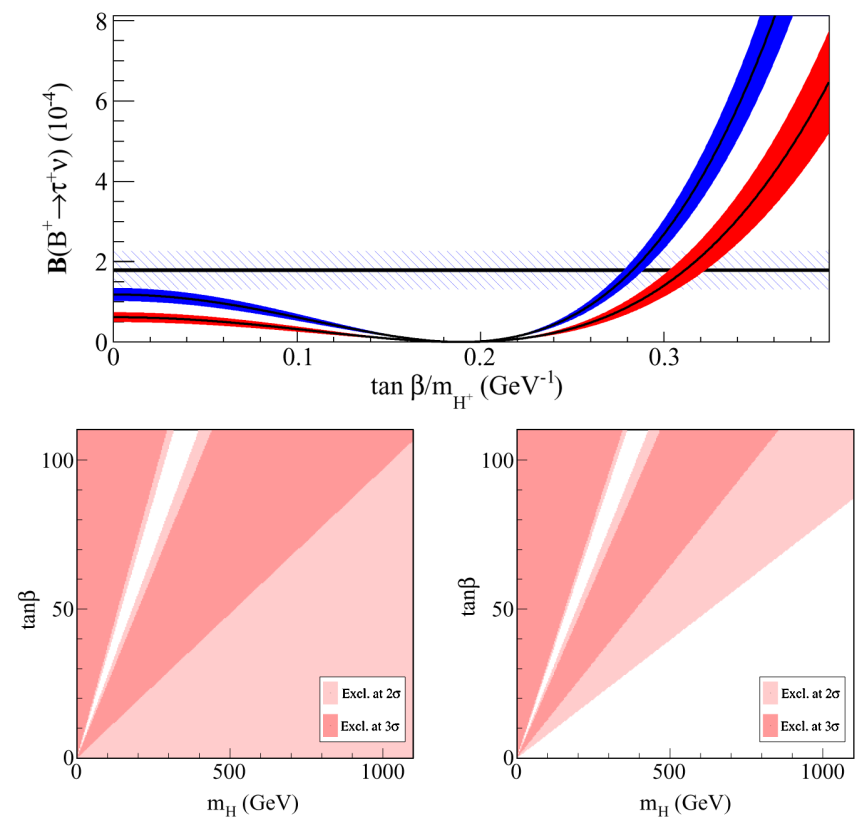

FIG. 4 (color online). Top plot: Comparison between the measured $\mathcal{B}\left(B^{+} \rightarrow \tau^{+} \nu\right)$ branching fraction (horizontal band) with the prediction of the $2 \mathrm{HDM}$ as a function of $\tan \beta / m_{H^{+}}$, using exclusive (red/light gray) or inclusive (blue/dark gray) $\left|V_{u b}\right|$ measurement. Bottom plots: $90 \%$ and $99 \%$ C.L. exclusion regions in the $\left(m_{H^{+}}, \tan \beta\right)$ plane using the exclusive (left) and inclusive (right) measurements of $\left|V_{u b}\right|$.

technique [12], and with the other measurement from Belle using semileptonic tag- $B \mathrm{~s}$ [13]. Combining this result with the other $B A B A R$ measurement of $\mathcal{B}\left(B^{+} \rightarrow \tau^{+} \nu\right)$ derived from a statistically independent sample [11], we obtain $\mathcal{B}\left(B^{+} \rightarrow \tau^{+} \nu\right)=(1.79 \pm 0.48) \times 10^{-4}$, where both statistical and systematic uncertainties are combined in quadrature.

Our measurement of the branching fraction $\mathcal{B}\left(B^{+} \rightarrow \tau^{+} \nu\right)$ exceeds the prediction of the SM determined using the values of $\left|V_{u b}\right|$ extracted from exclusive semileptonic events and from inclusive semileptonic events by $2.4 \sigma$ and $1.6 \sigma$, respectively. We also determine, separately for
PHYSICAL REVIEW D 88, 031102(R) (2013)

the exclusive and inclusive $\left|V_{u b}\right| B A B A R$ measurements, 90\% C.L. exclusion regions in the parameter space of the 2HDM- type II $\left(m_{H^{+}}, \tan \beta\right)$, where $m_{H^{+}}$is the charged Higgs mass and $\tan \beta$ is the ratio of the vacuum expectation values of the two Higgs doublets. We find that, taking $\left|V_{u b}\right|$ from the exclusive measurement, most of the parameters space is excluded at $90 \%$ C.L. Using the higher value of $\left|V_{u b}\right|$ from the inclusive measurement, the constraints are less stringent but already set a lower limit at the $\mathrm{TeV}$ scale for high $\tan \beta$. The same implications on 2HDM are supported by a recent $B A B A R$ study of the $\mathcal{B}\left(B \rightarrow D^{(*)} \tau \nu\right)$ decays [18]. Figure 4 shows a comparison between the measured $\mathcal{B}\left(B^{+} \rightarrow \tau^{+} \nu\right)$ branching fraction with the prediction of the 2HDM as a function of $\tan \beta / m_{H^{+}}$and the exclusion plots in the $\left(m_{H^{+}}, \tan \beta\right)$ plane for the exclusive and inclusive measurements of $\left|V_{u b}\right|$.

We are grateful for the extraordinary contributions of our PEP-II colleagues in achieving the excellent luminosity and machine conditions that have made this work possible. The success of this project also relies critically on the expertise and dedication of the computing organizations that support $B A B A R$. The collaborating institutions wish to thank SLAC for its support and the kind hospitality extended to them. This work is supported by the U.S. Department of Energy and National Science Foundation, the Natural Sciences and Engineering Research Council (Canada), the Commissariat à l'Energie Atomique and Institut National de Physique Nucléaire et de Physique des Particules (France), the Bundesministerium für Bildung und Forschung and Deutsche Forschungsgemeinschaft (Germany), the Istituto Nazionale di Fisica Nucleare (Italy), the Foundation for Fundamental Research on Matter (The Netherlands), the Research Council of Norway, the Ministry of Education and Science of the Russian Federation, Ministerio de Ciencia e Innovación (Spain), and the Science and Technology Facilities Council (United Kingdom). Individuals have received support from the Marie-Curie IEF program (European Union), the A. P. Sloan Foundation (USA) and the Binational Science Foundation (USA-Israel).
[1] Charge-conjugate modes are implied throughout the paper.

[2] N. Cabibbo, Phys. Rev. Lett. 10, 531 (1963); M. Kobayashi and T. Maskawa, Prog. Theor. Phys. 49, 652 (1973).

[3] H. Na, C. J. Monahan, C. T. H. Davies, R. Horgan, G. P. Lepage, and J. Shigemitsu (HPQCD Collaboration), Phys. Rev. D 86, 034506 (2012).

[4] J. P. Lees et al. (BABAR Collaboration), Phys. Rev. D 86, 092004 (2012).

[5] J. P. Lees et al. (BABAR Collaboration), Phys. Rev. D 86, 032004 (2012).
[6] W. S. Hou, Phys. Rev. D 48, 2342 (1993).

[7] A. G. Akeroyd and S. Recksiegel, J. Phys. G 29, 2311 (2003).

[8] B. Aubert et al. (BABAR Collaboration), Nucl. Instrum. Methods Phys. Res., Sect. A 479, 1 (2002); W. Menges, IEEE Nucl. Sci. Symp. Conf. Rec. 5, 1470 (2006).

[9] S. Agostinelli et al. (GEANT4 Collaboration), Nucl. Instrum. Methods Phys. Res., Sect. A 506, 250 (2003).

[10] B. Aubert et al. (BABAR Collaboration), Phys. Rev. D 77, 011107(R) (2008). 
EVIDENCE OF $B^{+} \rightarrow \tau^{+} \nu$ DECAYS...

[11] B. Aubert et al. (BABAR Collaboration), Phys. Rev. D 81, 051101(R) (2010).

[12] K. Hara et al. (Belle Collaboration), Phys. Rev. Lett. 110, 131801 (2013).

[13] K. Hara et al. (Belle Collaboration), Phys. Rev. D 82, 071101 (2010).

[14] H. Albrecht et al. (ARGUS Collaboration), Phys. Lett. B 185, 218 (1987).
PHYSICAL REVIEW D 88, 031102(R) (2013)

[15] M. J. Oreglia, Report No. SLAC-236, 1980; J.E. Gaiser, Report No. SLAC-255, 1982; T. Skwarnicki, Report No. DESY F31-86-02, 1986.

[16] G. C. Fox and S. Wolfram, Nucl. Phys. B149, 413 (1979).

[17] E. Farhi, Phys. Rev. Lett. 39, 1587 (1977).

[18] J. P. Lees et al. (BABAR Collaboration), Phys. Rev. Lett. 109101802 (2012). 